\title{
THERAPIES COGNITIVES DES DYSFONCTIONS SEXUELLES
}

\author{
Dr M. Bonierbale \\ Centre Médical Durandal \\ 21 Place A. Labadié - 13001 Marseille
}

\section{COGNITIVE THERAPY IN SEXUAL DYSFUNCTIONS. The basis of cognitive} therapy is an analysis of the way an individual thinks and perceives in order to analyse what will affect his feelings and behavior. The patterns of thoughts will determine in which way the ideas will be perceived and conceived. The author provides a list and, taking erectile dysfunction as a model, gives examples of dysfunctional schemas. Thus the essential points of the treatment are better known. This type is particularly interesting as it corresponds to the way the majority of patients think, and who can tackle it very easily. In our experience, $60 \%$ of patients choose a cognitive-behavioral therapy when offered a list of possible treatments for their dysfunction. This should be complementary to any therapy, even technical. Key words : Cognitive Therapy Patterns - Sexual Dysfunction. Andrologie, 1992, 2 : 82-83.

Les thérapies cognitives sont basées sur un ensemble de principes et de techniques thérapeutiques dont la structure théorique réside dans la psychologie cognitive, la théorie du traitement de l'information, et la psychologie sociale (1-7). La thérapie cognitive se base sur une analyse théorique de ce que la façon de penser et de percevoir chez un individu va influencer ses sentiments et son comportement. Ainsi, une situation perçue et interprétée comme dangereuse est génératrice d'anxiété et de comportement de fuite. Quant aux pensées et aux images mentales, ce sont des dérivés des croyances, des attitudes et des suppositions développées dans l'enfance.

Les problèmes psychologiques seraient alors causés par des processus communs tel que les fausses connaissances, les conclusions inexactes basées sur une information inadéquate ou inexacte, et l'incapacité de distinguer entre imagination et réalité.

L'individu qui a fait des suppositions erronées dans son enfance va alors formuler des règles ou des normes également erronées et rigides. Ces normes proviennent de ce que Beck (1) appelle schémas ou patterns complexes de pensée, qui vont déterminer de quelle façon les idées seront perçues et conçues.Ces patterns sont des espèces de moules perceptifs qui donnent forme aux données reçues. Ainsi la déformation du vécu peut-être maintenue grâce à des erreurs caractéristiques dans le traitement de l'information; ainsi des erreurs systématiques dans le raisonnement sont appelées des distorsions cognitives. On y retrouve :

- Les inférences arbitraires qui permettent d'arriver à des conclusions sans évidence pour les confirmer; ainsi une femme dont le mari a une dysérection peut juger: "il a sûrement une maîtresse"

- Les abstractions sélectives qui soustraient l'information de son contexte en soulignant certains détails.

- Dans la surgénéralisation, un incident isolé peut être interprété comme représentatif d'une situation semblable ; exemple, un homme ayant un échec sexuel avec une femme peut conclure: "je n'arrive à rien avec toutes les femmes".

- Dans la magnification et la minimisation, une circonstance est interprétée comme plus ou moins importante que la réalité; exemple, un homme ayant une petite perte d'érection arrive à la consultation d'un sexologue en disant: "je suis totalement impuissant".

- Dans la personnalisation, on peut s'attribuer des évènements externes sans qu'il y ait d'évidence pour en arriver à une telle conclusion ; par exemple, une femme qui voit son mari regarder une autre femme présume :" il ne me trouve plus séduisante",

- Dans la pensée dichotomique les expériences sont codifiées selon la loi du tout ou rien comme un succès fou ou un échec total, ce qui est particulièrement flagrant dans la population des hommes présentant des dysérections qui varient très souvent entre un rapport totalement réussi ou totalement nul.

- Quant à la désignation et aux fausses désignations, il s'agit d'un processus d'interprétation de sa propre identité à base d'imperfections et d'erreurs faites dans le passé.

Ce qu'il faut donc retenir, c'est que les schèmes sont des filtres perceptifs différents pour chaque individu, dépendant de ses interactions d'apprentissage avec le milieu extérieur, et qui sont des structures inconscientes sur lesquelles le sujet n'a pas de contrôle, et qui lui donnent sa vision du monde en acceptant, refusant, ou déformant les informations. Ces schèmes donnent à chaque personnalité son style et forment son système de croyance et ses attitudes. Ils se mettent en place pendant l'histoire des sujets, en réponse à ses expériences parentales existentielles, et ils peuvent être réactivés à chaque situation analogue. Ainsi un enfant qui n'a pas eu une relation affective satisfaisante avec ses parents pourra croire: “je ne suis pas digne d'attention, il faut que je rachète ma faute" ou bien "je ne vaut rien et personne ne peut s'attacher à moi"; schème qui peut être réactivé sous la forme de sentiments de culpabilité ou de tristesse lors de sentiments de frustration affective ou d'une séparation.

\section{ASPECTS COGNITIFS DE LA SEXUALITÉ DANS UNE PERSPECTIVE COGNITIVE DES DYSFONCTIONS SEXUELLES}

Il est intéressant, avant tout lors des consultations, de permettre au patient de repérer l'affect qui déforme sa perception (le plus souvent de l'anxiété ou de la culpabilité) et le schème qui y est attaché (le script). Ainsi on trouve souvent :

- Je suis incompétent, pas à la hauteur; "Je ne vais pas y arriver".

- Je ne suis pas digne d'intérêt, d'amour; "Cette femme me trompe sûrement"

- Je n'apporte pas à ma partenaire ce qu'elle mérite; "Je n'ai pas la performance sexuelle qu'elle est en droit d'attendre de moi"

- Je vais être mal jugé et ridicule; "Je ne sais pas m'y prendre et je suis mal fait"

- Elle ne vaut pas la peine qu'on s'interesse à elle; "Je ne m'attacherai pas"

- Le monde est plein de dangers, de pollution : "Je risque d'attraper une MST en ayant des rapports sexuels" etc..

Si on prend pour modèle la dysérection : une érection nécessite que les voies excito-motrices du réflexe érectile soient en bon état de marche sur le plan neuro-vasculaire et tissulaire, et le schème du scénario excito-moteur de ces voies également. La distorsion du schème de mise en route du réflexe érectile peut s'accompagner d'une réaction d'anxiété ou d'une absence d'excitation, ou d'une réaction aversive qui soit inhibe soit inverse le schème excito-moteur du reflexe érectile. Ainsi, dans les affects anxieux, on peut avoir le script: "Je dois avoir une érection dès que ma partenaire en a le désir. Je n'y 
arriverai jamais", alors que dans l'aversion on retrouve le script: "Cette femme a l'air d'avoir une mauvaise vie, je crains d'attraper le Sida". Lorsqu'il n'y a pas de filtre émotif prédominant, et qu'il y a des postulats automatiques avant tout, on peut retrouver différents modèles comme :

\section{- Donnant-donnant :}

"Je rapporte de l'argent à la maison, ma femme me doit des rapports sexuels"

"Ma femme prend soin de moi chaque jour, je lui dois des rapports quotidiens"

\section{Devoir conjugal.}

"Je ne remplis pas mon devoir conjugal, je dois divorcer"

- Contrat sexuel.

" Ma femme peut prendre des amants puisque je ne satisfais pas ses besoins sexuels"

\section{LA PRISE EN CHARGE}

Quelles que soient les modalités et les variations du dysfonctionnement perceptif, la prise en charge necessite un programme :

1 - d'information, destiné à la correction des conceptualisations erronées.

2 - de dédramatisation, afin de réduire l'anxiété responsable des conduites d'évitement et de l'inhibition du schème excito-moteur réflexe de l'érection, ou de déculpabilisation, si c'est la culpabilité qui est au devant de la scène ou d'autorisation et d'affirmation de soi si c'est l'inhibition qui domine.

3 - de présentation d'autres modes perceptifs afin d'arriver à un changement du mode de pensée permettant de repérer que ce n'est pas l'érection qui est malade mais sa mise en route.

Ainsi une désensibilisation in-vivo avec injections intra-caverneuses de soutien, de vasodilatateur, vise à rétablir les schèmes comportementaux dissociés des schèmes dysfonctionnels d'échec, et permet de repérer que ce n'est pas l'érection qui est en cause.

4 - adopter des stratégies de solution de problème visant à rétablir l'excitation; comme le classique sensate-focus, comme la relaxation et la désensibilisation en imagination avec injection de fantasmes qui stimulent les images positives et éteignent l'inhibition anxieuse qui entretiennent le cercle vicieux dysfonctionnel.

5 - Une évaluation des évènements déclenchants directs ou indirects renseigne également sur le fonctionnement du schème erroné dysfonctionel et permet de le pointer.

En somme, il faut permettre au patient de prendre conscience des pensées automatiques négatives qui déforment ses perceptions et entrânent des comportements erronés, lui faire reconnaître la liaison qu'il y a entre les pensées, l'affect, et le comportement dysfonctionnel, lui faire examiner l'évidence qu'il y a pour et contre ses pensées automatiques erronées, lui substituer des interprétations plus réalistes, et se servir de consignes comportementales pour tester les hypothèses erronées.

\section{RESULTATS ET DISCUSSIONS}

Dans notre expérience personnelle, $60 \%$ des patients a qui on propose une liste de possibilités de prise en charge d'une dysfonction sexuelle, choisissent une thérapie cognitivo-comportementale, et $10 \%$ s'arrêtent en cours de traitement. Les critères de guérison sont la décristallisation du symptôme et le sentiment de satisfaction face au changement de comportement ou au changement de système perceptif.

La limite scientifique de l'évaluation de ces thérapies, comme de toutes thérapies psychologiques, vient de ce qu'aucune évaluation scientifique ne permet de comparer les systèmes thérapeutiques psychologiques entre eux et aux autres prises en charge mixtes, si bien que quelles que soient les méthodes utilisées, il semble que ce soit plutôt celle que choisit le patient qui donne les meilleurs résultats, car elle correspond à sa motivation. Or la motivation intervient pour $60 \%$ dans ce qui fait marcher une thérapie, alors que d'après les travaux de Guerin la personnalité et le savoir faire du thérapeute n'interviennent que pour $30 \%$ et la technique thérapeutique pour $10 \%$.

Dans la littérature, il y a peu de résultats évalués pour les thérapies cognitivo-comportementales. Obler (8), en 1973, compare cette approche à celle d'une psychanalyse et d'une liste d'attente dite de "contrôle" pour des cas de dysérection et d'éjaculation prématurées et retrouve les chiffres suivants :

$80 \%$ de bons résultats pour les thérapies cognitivo-comportementales,

$10 \%$ pour les thérapies analytiques,

$10 \%$ pour le groupe contrôle.

\section{CONCLUSION}

De nos jours, face à l'évolution des systèmes de pensée humains, il semble que les apports cognitifs aient fait évoluer les psychothérapies dans la mesure où ils sont plus en accord avec les systèmes de pensée actuels, et de ce fait plus à la portée des patients et même des thérapeutes.

Mais de toute façon, chacun doit savoir rester modeste et savoir que:

-quel que soit le mode thérapeutique mis en jeu lorsqu'on intervient sur quelque chose d'aussi complexe que la sexualité

-que son abord soit fait par le corps malade, par l'esprit, ou par la relation au partenaire, on ne sait pas grand chose sur ce qu'il convient de faire et de croire, et que tous les systèmes thérapeutiques ne peuvent qu'être adaptés à ce que peut le patient, et à ce qu'il veut réellement changer.
C'est donc vers cela que doivent tendre les évaluations thérapeutiques, plutôt que sur la cristallisation sur un seul symptôme qui n'est qu'un indicateur sinon un paravent. Sinon sous des allures de repérage scientifique, on risque de ne faire en toute bonne foi que du scientisme:

\section{RÉFÉRENCES}

1- Beck A. Cognitive therapy and the emotional disorders. International University Press, New York, 1976.

2 - Dattilio F. Thérapie cognitive de couple. Journal de Thérapie Comportementale et Cognitive, 1992 , 2: $17-24$.

3 - Ellis A. The nature of disturbed marital interactions. In: Ellis A. et Greiger R. eds. Handbook of rational emotive therapy. Springer, New York, 1977, 170-176.

4 - Epstein N. Cognitive marital therapy : a multilevel assessment and intervention. Journal of rational emotive therapy, 1986, 4: 68-81.

5 - Freeman A, Greenwood V. Cognitive therapy: applications in psychiatric and medical settings. S. Human sciences press, New York, 1987.

6 - Jacobsen N. The modification of cognitive processes in behavioral marital therapy : integrating cognitive and behavioral intervention strategies. In: Marital interaction: analysis and modification, Hahlweg K et Jacobsen N eds, Guilford, New York, 1984.

7 - Kendall P, Hollon S. Cognitive behavioral interventions: theory, research and procedures. Academic Press, New York, 1979.

8 - Obler M. Systematic desensitization in sexual disorders. J. Behav. Ther. Exp. Pychiat., 1973, 4: 93-1010.

RESUME : Les bases de la thérapie cognitive font analyser la façon de penser et de percevoir d'un individu afin d'analyser ce qui va effleurer ses sentiments et son comportement. Ces points sont très importants dans toutes les dysfunctions sexuelles. Les patterns de pensées vont déterminer de quelle façon les idées seront perçues et conçues. L'auteur en fait une liste, et prenant comme modèle la dysérection, donne des exemples de schèmes dysfonctionnels. Ceci permet de mieux comprendre quels sont les points essentiels de la prise en charge. Ce type d'abord est particulièrement intéressant car il correspond au système de pensée de la majorité des patients, qui peuvent l'aborder très facilement. Dans notre expérience, 60 $\%$ des patients choisissent une thérapie cognitive-comportementale lorsqu' on leur propose une liste de possibilité de prises en charges de leur dysfunction. Elles doivent pouvoir complémenter nimporte quel geste thérapeutique, même technique. Mots clés : Thérapie Cognitive - Patterns - Dysfonction Sexuelle. Andrologie, 1992, 2 : 82-83. 